\title{
Landau and Kolmogoroff Type Polynomial Inequalities
}

\author{
CLAUDIA R.R. ALVES and DIMITAR K. DIMITROV*
}

Departamento de Ciências de Computação e Estatística, IBILCE, Universidade Estadual Paulista, 15054-000 São José do Rio Preto, SP, Brazil

(Received 4 December 1998; Revised 2 February 1999)

Let $0<j<m \leq n$ be integers. Denote by $\|\cdot\|$ the norm $\|f\|^{2}=\int_{-\infty}^{\infty} f^{2}(x) \exp \left(-x^{2}\right) \mathrm{d} x$. For various positive values of $A$ and $B$ we establish Kolmogoroff type inequalities

$$
\left\|f^{(j)}\right\|^{2} \leq \frac{A\left\|f^{(m)}\right\|+B\|f\|}{A \theta_{k}+B \mu_{k}},
$$

with certain constants $\theta_{k} \mathrm{e} \mu_{k}$, which hold for every $f \in \pi_{n}$ ( $\pi_{n}$ denotes the space of real algebraic polynomials of degree not exceeding $n$ ).

For the particular case $j=1$ and $m=2$, we provide a complete characterisation of the positive constants $A$ and $B$, for which the corresponding Landau type polynomial inequalities

$$
\left\|f^{\prime}\right\| \leq \frac{A\left\|f^{\prime \prime}\right\|+B\|f\|}{A \theta_{k}+B \mu_{k}}
$$

hold. In each case we determine the corresponding extremal polynomials for which equalities are attained.

Keywords: Landau and Kolmogoroff type inequalities; Markov's inequality; Hermite polynomials; Extremal polynomials; Rayleigh-Ritz theorem

1991 Mathematics Subject Classification: Primary 41A17, 26D15; Secondary 33C45

*Corresponding author. E-mail: dimitrov@nimitz.dcce.ibilce.unesp.br. 


\section{INTRODUCTION AND STATEMENT OF RESULTS}

Let $\|f\|_{\infty,[a, b]}=\sup _{a \leq x \leq b}|f(x)|$. In 1913, Landau [4] proved that $\left\|f^{\prime}\right\|_{\infty,[0,1]} \leq 4$ for every $f \in C^{2}[0,1]$, for which $\|f\|_{\infty,[0,1]}=1$ and $\left\|f^{\prime \prime}\right\|_{\infty,[0,1]}=4$. Kolmogoroff [3] proved, for sufficiently smooth functions, inequalities of the form

$$
\left\|f^{(j)}\right\|_{\infty,[0,1]} \leq K(m, j)\left\|f^{(m)}\right\|_{\infty,[0,1]}^{j / m}\|f\|_{\infty,[0,1]}^{1-(j / m)}
$$

with the best constant $K(m, j)$ and determined the functions for which inequality in (1) is attained. These extremal functions are perfect splines and in none of the cases algebraic polynomials. On the other hand, the classical A. Markov's inequality [5]

$$
\left\|p^{\prime}\right\|_{\infty,[-1,1]} \leq n^{2}\|p\|_{\infty,[-1,1]}, \quad p \in \pi_{n}
$$

and its extension,

$$
\left\|p^{(k)}\right\|_{\infty,[-1,1]} \leq \frac{1}{(2 k-1) ! !} \prod_{i=0}^{k-1}\left(n^{2}-i^{2}\right)\|p\|_{\infty,[-1,1]}, \quad 1 \leq k \leq n, \quad p \in \pi_{n}
$$

given by V. Markov [6], are typical examples of inequalities connecting norms of derivatives of different orders of polynomials.

These facts motivated some author $[1,8]$ to look for polynomial analogues of Landau's and Kolmogoroff's inequalities. In particular, Varma [8] established a sharp Landau type inequality and in a recent paper Bojanov and Varma [1] proved a Kolmogoroff type polynomial inequality for the weighted norm $\|f\|^{2}=\int_{-\infty}^{\infty} f^{2}(x) \exp \left(-x^{2}\right) \mathrm{d} x$. The extremal polynomials for which the inequalities in [8] and [1] reduce to equalities are the classical Hermite polynomials $H_{n}(x)$, orthogonal on $(-\infty, \infty)$ with respect to the weight function $\exp \left(-x^{2}\right)$.

In this paper we suggest a somehow more systematic approach than the one developed in [1], which allows us to establish the following Kolomogoroff type weighted polynomial inequalities.

THEOREM 1 Let $j<m \leq n$ be the positive integers and $A$ and $B$ positive constants. 
(i) If

$$
\frac{A}{B} \leq 2^{-m} \frac{(n-m) !}{n !} \frac{j}{m-j}
$$

then

$$
\left\|f^{(j)}\right\|^{2} \leq \frac{A\left\|f^{(m)}\right\|^{2}+B\|f\|^{2}}{A 2^{m-j}(n-j) ![(n-m) !]^{-1}+B 2^{-j}(n-j) !(n !)^{-1}}
$$

for every $f \in \pi_{n}$. Moreover, equality is attained if and only if $f(x)$ is a constant multiple of $H_{n}(x)$.

(ii) If

$$
\frac{2^{-m}}{(m+1) !} \frac{j}{(m-j)} \leq \frac{A}{B} \leq 2^{-m} \frac{j}{m !(m-j)}
$$

then

$$
\left\|f^{(j)}\right\|^{2} \leq \frac{A\left\|f^{(m)}\right\|^{2}+B\|f\|^{2}}{A 2^{m-j}(m-j) !+B 2^{-j}(m-j) !(m !)^{-1}}
$$

for every $f \in \pi_{n}$. Moreover, equality is attained if and only if $f(x)$ is a constant multiple of $H_{m}(x)$.

(iii) If

$$
\frac{A}{B}>2^{-m} \frac{j}{m !(m-j)}
$$

then

$$
\left\|f^{(j)}\right\|^{2} \leq \frac{A\left\|f^{(m)}\right\|^{2}+B\|f\|^{2}}{B 2^{-j}(m-j-1) ![(m-1) !]^{-1}}
$$

for every $f \in \pi_{n}$. Moreover, equality is attained if and only if $f(x)$ is a constant multiple of $H_{m-1}(x)$.

(iv) If $A / B=2^{-m} j /((m-j) m !)$, then the inequalities (4) and (5) coincide and they hold for every $f \in \pi_{n}$. In this case equality is attained if and only if $f(x)$ is any linear combination of $H_{m-1}(x)$ and $H_{m}(x)$. 
As an immediate consequence of Theorem 1(i) we obtain COROLlary 1 Let

$$
\alpha \leq \frac{(n-m) ! j}{2^{m-j}(n-j) ! m} .
$$

Then the inequality

$$
\left\|f^{(j)}\right\|^{2} \leq \alpha\left\|f^{(m)}\right\|^{2}+\left\{2^{j}\left(\begin{array}{c}
n \\
j
\end{array}\right) j !-\alpha 2^{m}\left(\begin{array}{c}
n \\
m
\end{array}\right) m !\right\}\|f\|^{2}
$$

holds for every $f \in \pi_{n}$. Moreover, equality is attained if and only if $f(x)$ is a constant multiple of $H_{n}(x)$.

This is exactly the result of Bojanov and Varma [1] mentioned above.

In this case $j=1$ and $m=2$ we provide a complete characterisation of the positive constants $A$ and $B$, for which the corresponding Landau type polynomial inequalities hold.

THEOREM 2 Let $A$ and $B$ be positive constants.

(i) If $0<A / B<(4 n(n-1))^{-1}$, then

$$
\left\|f^{\prime}\right\|^{2} \leq \frac{A}{2 A(n-1)+B(2 n)^{-1}}\left\|f^{\prime \prime}\right\|^{2}+\frac{B}{2 A(n-1)+B(2 n)^{-1}}\|f\|^{2},
$$

for every $f \in \pi_{n}$. Moreover, equality is attained if and only if $f(x)=$ $c H_{n}(x)$, where $c$ is a constant.

(ii) If $(4 k(k+1))^{-1}<A / B<(4 k(k-1))^{-1}$, where $k \in \mathbb{N}, 2<k<n-1$, then

$$
\left\|f^{\prime}\right\|^{2} \leq \frac{A}{2 A(k-1)+B(2 k)^{-1}}\left\|f^{\prime \prime}\right\|^{2}+\frac{B}{2 A(k-1)+B(2 k)^{-1}}\|f\|^{2},
$$

for every $f \in \pi_{n}$. Moreover, equality is attained if and only if $f(x)=$ $c H_{k}(x)$, where $c$ is a constant. 
(iii) If $1 / 8<A / B<\infty$, then

$$
\left\|f^{\prime}\right\|^{2} \leq \frac{2 A}{B}\left\|f^{\prime \prime}\right\|^{2}+2\|f\|^{2}
$$

for every $f \in \pi_{n}$. Moreover, equality is attained if and only if $f(x)=$ $c H_{1}(x)$.

(iv) If $A / B=(4 k(k+1))^{-1}$ for some integer $k$, then the inequalities

$$
\left\|f^{\prime}\right\|^{2} \leq \frac{A}{2 A(k-1)+B(2 k)^{-1}}\left\|f^{\prime \prime}\right\|^{2}+\frac{B}{2 A(k-1)+B(2 k)^{-1}}\|f\|^{2},
$$

and

$$
\left\|f^{\prime}\right\|^{2} \leq \frac{A}{2 A k+B[2(k+1)]^{-1}}\left\|f^{\prime \prime}\right\|^{2}+\frac{B}{2 A k+B[2(k+1)]^{-1}}\|f\|^{2},
$$

coincide and they hold for every $f \in \pi_{n}$. In this case equality in (9) and (10) is attained if and only if $f(x)$ is any linear combination of $H_{k}(x)$ and $H_{k+1}(x)$.

Setting $B=4 n^{2} A$, in Theorem 3(i) we obtain the inequality

$$
\left\|f^{\prime}\right\|^{2} \leq \frac{1}{2(2 n-1)}\left\|f^{\prime \prime}\right\|^{2}+\frac{2 n^{2}}{2 n-1}\|f\|^{2}, \quad f \in \pi_{n}
$$

where equality is attained only for the polynomials $f(x)$ that are constant multiples of $H_{n}(x)$. This is nothing but Varma's result [8].

\section{A PRELIMINARY RESULT}

Our idea is to study, for any given integers $j, m, n, 0<j<m \leq n$, and positive constants $A$ and $B$, the extremal problem

$$
\min \left\{\frac{A\left\|f^{(m)}\right\|^{2}+B\|f\|^{2}}{\left\|f^{(j)}\right\|^{2}}: f \in \pi_{n}, f(x) \neq 0\right\},
$$


where the objective function $F(A, B, f)=\left(A\left\|f^{(m)}\right\|^{2}+B\|f\|^{2}\right) /\left\|f^{(j)}\right\|^{2}$ depends on the parameters $A$ and $B$. Denote by $f_{*} \in \pi_{n}$ the extremal polynomial, that is, the polynomial for which the minimum of $F(A, B, f)$ is attained. Thus we define

$$
F(A, B)=F\left(A, B, f_{*}\right)=\min \left\{F(A, B, f): f \in \pi_{n}, f(x) \neq 0\right\} .
$$

Let the sequences $\left\{\mu_{i}\right\}_{i=j}^{n},\left\{\theta_{i}\right\}_{i=m}^{n}$ and $\left\{\gamma_{k}\right\}_{k=j}^{n}$ be defined by

$$
\begin{aligned}
\mu_{i} & =2^{-j} \frac{(i-j) !}{i !}, \quad \theta_{i}=2^{m-j} \frac{(i-j) !}{(i-m) !}, \\
\gamma_{k} & =B \mu_{k}, \quad k=j, \ldots, m-1, \\
\gamma_{k} & =A \theta_{k}+B \mu_{k}, \quad k=m, \ldots, n .
\end{aligned}
$$

LEMMA 1 For any given integers $j<m \leq n$ and positive constants $A$ and $B$

$$
F(A, B)=\min _{j \leq i \leq n} \gamma_{i}:=\gamma_{k}
$$

Proof We need two basic properties of the Hermite polynomials $H_{n}$ (cf. (5.5.1) and (5.5.10) in [7]):

$$
\int_{-\infty}^{\infty} H_{k}(x) H_{i}(x) \exp \left(-x^{2}\right) \mathrm{d} x=\sqrt{\pi} 2^{k} k ! \delta_{i k},
$$

where $\delta_{i k}$ is the Kronecker delta, and

$$
H_{i}^{\prime}(x)=2 i H_{i-1}(x) .
$$

In what follows a different normalisation of the Hermite polynomials will be used. Set $\tilde{H}_{i}(x)=c_{i} H_{i}(x)$, where

$$
c_{i}=1 \text { for } i=0, \ldots, j-1,
$$

and

$$
c_{i}=\left(\sqrt{\pi} 2^{i+j} \frac{(i !)^{2}}{(i-j) !}\right)^{-1 / 2} \quad \text { for } i=j, \ldots, n
$$


Since $\left\{H_{n}\right\}$ are orthogonal, the polynomials $\tilde{H}_{0}(x), \ldots, \tilde{H}_{n}(x)$ form a basis in $\pi_{n}$. Then every $f \in \pi_{n}$ can be uniquely represented as a linear combination $f(x)=\sum_{k=0}^{n} a_{k} \tilde{H}_{k}(x)$. Hence the orthogonality relation (12) and the definition of the polynomials $\tilde{H}_{i}(x)$ yield

$$
\|f\|^{2}=\int_{-\infty}^{\infty}\left(\sum_{i=0}^{n} a_{i} \tilde{H}_{i}(x)\right)^{2} \exp \left(-x^{2}\right) \mathrm{d} x=\sum_{i=0}^{n} \mu_{i} a_{i}^{2},
$$

where $\mu_{i}=\sqrt{\pi} 2^{i} i$ ! for $i=0, \ldots, j-1$ and $\mu_{i}$ for $i=j, \ldots, n$ are defined above.

Similarly, the relations (12), (13) and the definition of $\tilde{H}_{i}(x)$ imply

$$
\left\|f^{(j)}\right\|^{2}=\sum_{i=j}^{n} a_{i}^{2} c_{i}^{2}\left\|H_{i}^{(j)}\right\|^{2}=\sum_{i=j}^{n} a_{i}^{2}
$$

In the same manner we obtain

$$
\left\|f^{(m)}\right\|^{2}=\sum_{i=m}^{n} \theta_{i} a_{i}^{2}
$$

where $\theta_{i}, i=m, \ldots, n$, are defined above.

Thus the problem formulated in the beginning of this section reduces to the following one:

$$
\min \left\{\left(\sum_{i=0}^{n} B \mu_{i} a_{i}^{2}+\sum_{i=m}^{n}\left(A \theta_{i}+B \mu_{i}\right) a_{i}^{2}\right) / \sum_{i=j}^{m} a_{i}^{2}: a_{0}, \ldots, a_{n} \in \mathbb{R}\right\}
$$

Obviously the above minimum is attained for $a_{0}=\cdots=a_{j-1}=0$, that is,

$$
\begin{aligned}
& F(A, B) \\
& \quad=\min \left\{\sum_{i=j}^{n} B \mu_{i} a_{i}^{2}+\sum_{i=m}^{n}\left(A \theta_{i}+B \mu_{i}\right) a_{i}^{2}: a_{j}, \ldots, a_{n} \in \mathbb{R}, \sum_{i=j}^{n} a_{i}^{2}=1\right\} .
\end{aligned}
$$


Let $\bar{a}=\left(a_{j}, \ldots, a_{n}\right)$. Therefore our problem reduces to determine the minimum of $\bar{a}^{t} C \bar{a}$, subject to $\bar{a}^{t} \bar{a}=1$, where $C$ is the diagonal matrix

$$
\operatorname{diag}\left(B \mu_{j}, \ldots, B \mu_{m-1}, A \theta_{m}+B \mu_{m}, \ldots, A \theta_{n}+B \mu_{n}\right) .
$$

By the Rayleigh-Ritz Theorem (cf. Theorem 4.2.2 on p. 176 in Horn and Johnson [2]), $F(A, B)$ is equal to the smallest eigenvalue of $C$, that is,

$$
F(A, B)=\min \left\{\gamma_{j}, \ldots, \gamma_{n}\right\} .
$$

Moreover, if $F(A, B)=\gamma_{k}$, the extremal polynomial $f_{*}(x)$, for which $F(A, B)=F\left(A, B, f_{*}\right)$, is a constant multiple of $H_{k}(x)$.

\section{PROOFS OF THE THEOREMS}

Proof of Theorem 1 The sequence $\mu$ is decreasing. Indeed,

$$
\mu_{i}-\mu_{i+1}=2^{-j}\left(\frac{(i-j) !}{i !}-\frac{(i-j+1) !}{(i+1) !}\right)=2^{-j} \frac{(i-j) ! j}{(i+1) !} \geq 0 .
$$

Then the smallest among the numbers $B \mu_{j}, \ldots, B \mu_{m-1}$ is $B \mu_{m-1}$. Thus, according to Lemma 1 , we need to find the smallest $\gamma_{\nu}$ among $\gamma_{m}, \ldots, \gamma_{n}$ and to compare $\gamma_{\nu}$ with $B \mu_{m-1}$.

Consider the monotonicity of the sequence $\left\{\gamma_{k}\right\}_{k=m}^{n}$. Since

$$
\gamma_{k+1}-\gamma_{k}=A\left(\theta_{k+1}-\theta_{k}\right)+B\left(\mu_{k}-\mu_{k+1}\right),
$$

then (a) $\left\{\gamma_{k}\right\}_{k=m}^{n}$ is increasing if $A / B \geq\left(\mu_{k}-\mu_{k+1}\right) /\left(\theta_{k+1}-\theta_{k}\right)=: S_{k}$ for $k=m, \ldots, n-1$ and (b) $\left\{\gamma_{k}\right\}_{k=m}^{n}$ is decreasing if $A / B \leq S_{k}$ for $k=m, \ldots, n-1$.

Straightforward calculations show that

$$
S_{k}=\frac{\mu_{k}-\mu_{k+1}}{\theta_{k+1}-\theta_{k}}=2^{-m} \frac{(k-m+1) !}{(k+1) !} \frac{j}{m-j},
$$

and then $S_{k+1} / S_{k}=(k-m+2) /(k+2)<1$. This means that $\left\{S_{k}\right\}$ is a decreasing sequence. Hence, if $A / B \geq S_{m}$, then, $\gamma_{k}$ is increasing and then $\gamma_{\nu}=\gamma_{m}$. Thus, in this case we have

$$
F(A, B)=\min _{j \leq k \leq n} \gamma_{k}=\min \left\{\gamma_{m-1}, \gamma_{m}\right\}
$$


In order to compare $\gamma_{m-1}$ and $\gamma_{m}$, observe that $\gamma_{m-1}<\gamma_{m}$ if $\left(\mu_{m-1}-\mu_{m}\right) /$ $\theta_{m}<A \mid B$ and $\gamma_{m-1} \geq \gamma_{m}$ otherwise. In view of the identity $\left(\mu_{m-1}-\mu_{m}\right) /$ $\theta_{m}=2^{-m} j /((m-j) m !)$ we can conclude:

(1) If $A / B \geq S_{m}$ and $A / B>2^{-m} j /\left((m-j) m\right.$ !), then $\gamma_{m-1}<\gamma_{m}$ and $F(A, B)=\gamma_{m-1}$.

(2) If $S_{m} \leq A / B<2^{-m} j /\left((m-j) m\right.$ !), then $\gamma_{m-1}>\gamma_{m}$ and $F(A, B)=\gamma_{m}$.

It is worth mentioning that the interval $\left[S_{m}, 2^{-m} j /((m-j) m !)\right]$ is not empty because the inequality $S_{m}<2^{-m} j /((m-j) m !)$ is equivalent to the obvious one $m>0$.

The latter cases (1) and (2) correspond to the statements (iii) and (ii) of Theorem 1.

The above observation (b) and the monotonicity of $S_{k}$ imply that the sequence $\left\{\gamma_{k}\right\}_{k=m}^{n}$ is decreasing provided $A / B \leq S_{n-1}$. Hence, in this case we have $F(A, B)=\min \left\{\gamma_{m-1}, \gamma_{n}\right\}$. In order to compare $\gamma_{m-1}$ and $\gamma_{n}$, note that $\gamma_{m-1}<\gamma_{n}$ if $\left(\mu_{m-1}-\mu_{n}\right) / \theta_{n}<A / B$ and $\gamma_{m-1} \leq \gamma_{n}$ otherwise.

In view of the identity

$$
\frac{\mu_{m-1}-\mu_{n}}{\theta_{n}}=2^{-m} \frac{(n-m) !}{(n-j) !} \frac{[(m-j-1) ! n !-(n-j) !(m-1) !]}{(m-1) ! n !},
$$

we need a relation between the latter expression and $A / B$. On the other hand, the inequality

$$
\left(\begin{array}{c}
m \\
j
\end{array}\right)<\left(\begin{array}{c}
n \\
j
\end{array}\right) \text { for } j<m<n
$$

yields

$$
S_{n-1}<2^{-m} \frac{(n-m) !}{(n-j) !} \frac{[(m-j-1) ! n !-(n-j) !(m-1) !]}{(m-1) ! n !},
$$

which means that $\left(\mu_{m-1}-\mu_{n}\right) / \theta_{n}<S_{n-1}$. If

$$
\frac{A}{B} \leq S_{n-1}
$$

and

$$
\frac{A}{B}<2^{-m} \frac{(n-m) ![(m-j-1) ! n !-(n-j) !(m-1) !]}{(n-j) !}
$$


then $\gamma_{m-1}>\gamma_{n}$ and $F(A, B)=\gamma_{n}$. This corresponds to the statement (i) of the theorem.

Proof of Theorem 2 Since $j=1$ and $m=2$, Lemma 1 shows that we need to determine

$$
\min \left\{B \mu_{1}, A \theta_{2}+B \mu_{2}, \ldots, A \theta_{n}+B \mu_{n}\right\} .
$$

In order to this, we shall find the smallest among the numbers $A \theta_{2}+B \mu_{2}, \ldots, A \theta_{n}+B \mu_{n}$, say $\gamma_{\nu}$, and in each case we shall compare $\gamma_{\nu}$ to $B \mu_{1}$.

In what follows, up to the final observation in this proof, we shall assume that $2^{m} A+B=1$. Then we have

$$
\gamma_{k}=A \theta_{k}+B \mu_{k}=(B / k+(1-B)(k-1)) / 2 \text { for } k=2, \ldots, n \text {. }
$$

Define the function

$$
g(x)=(B / x+(1-B)(x-1)) / 2 \text { for } 2 \leq x \leq n .
$$

Since $g(k)=\gamma_{k}$ for $k=2, \ldots, n$, then our problem reduces to investigate the behaviour of $g(x)$ when $A$ and $B$ belong to the segment $2^{m} A+B=1$, $A, B>0$. Note that $2 g^{\prime}(x)=-B / x^{2}+(1-B)=0$, if and only if $x=$ $\pm(B /(1-B))^{1 / 2}$ and $g^{\prime \prime}(x)=B / x^{3}>0$ for $x>0$. Hence $g(x)$ is convex on the positive half-line and it attains its absolute minimum there at $x=$ $(B /(1-B))^{1 / 2}$. Thus, we can conclude that:

- If $\sqrt{B /(1-B)}<2$, then $\gamma_{\min }=\gamma_{2}$.

- If $\sqrt{B /(1-B)}>n$, then $\gamma_{\min }=\gamma_{n}$.

- If $k \leq \sqrt{B /(1-B)}<k+1$, where $2 \leq k<n-1$, then $\gamma_{\nu}=$ $\min \left\{\gamma_{k}, \gamma_{k+1}\right\}$.

In order to determine the smaller among $\gamma_{k}$ and $\gamma_{k+1}$, observe that

$$
\gamma_{k+1}<\gamma_{k} \text { if } \frac{k(k+1)}{k^{2}+k+1}<B
$$

and $\gamma_{k+1} \geq \gamma_{k}$ otherwise. It is clear that $\gamma_{k}=\gamma_{k+1}$ if and only if $B=k(k+1) /\left(k^{2}+k+1\right)$. 
Set $y:=(B /(1-B))^{1 / 2}$ for any $B, 0 \leq B<1$. If $B=k(k+1) /\left(k^{2}+k+1\right)$ the point of minimum of $g(x)$ is

$$
y_{k}:=\sqrt{k(k+1)}
$$

Obviously $k<y_{k}<k+1$. Since the function $g(x)$ is convex, then $\gamma_{\nu}=\gamma_{k+1}$ if and only if $y_{k}<y<y_{k+1}$ and this conclusion holds for $k=1, \ldots, n-2$. The latter inequality is itself equivalent to

$$
\frac{k(k+1)}{k^{2}+k+1}<B<\frac{(k+1)(k+2)}{k^{2}+3 k+3} .
$$

Let us compare, in each of these cases, $\gamma_{k+1}$ to $\gamma_{1}=B / 2$.

For $B \in(0,6 / 7)$, we need to compare $\gamma_{1}$ and $\gamma_{2}$. Since $\gamma_{2}-\gamma_{1}=$ $(1-3 B / 2) / 2$, then $\gamma_{1}<\gamma_{2}$ for $0<B<2 / 3, \gamma_{1}=\gamma_{2}$ for $B=2 / 3$ and $\gamma_{2}<\gamma_{1}$ for $2 / 3<B<6 / 7$.

Let now $k$ be any integer, such that $2 \leq k \leq n$ and let

$$
B \in\left(\frac{k(k+1)}{k^{2}+k+1}, \frac{(k+1)(k+2)}{k^{2}+3 k+3}\right)=\Delta_{k} .
$$

Since

$$
\gamma_{k+1}-\gamma_{1}=\frac{k}{2(k+1)}((k+1)-2 B(k+2)) \leq 0
$$

if and only if $(k+1) /(k+2)<B$ and this latter inequality always holds for $B \in \Delta_{k}$, then we have $\gamma_{k+1}<\gamma_{1}$ for every $B \in \Delta_{k}$.

Finally, we have $\gamma_{n}<\gamma_{1}$ for every

$$
B \in\left(\frac{n(n-1)}{n^{2}-n+1}, 1\right)=\Delta_{n-1}
$$

because $\gamma_{n}-\gamma_{1}=(n-1)(n-B(n+1)) / n<0$ is equivalent to $n /$ $(n+1)<B$ and obviously $n /(n+1)<n(n-1) /\left(n^{2}-n+1\right)$.

Recall that all considerations have been done under the restriction $2^{m} A+B=1$. The restrictions $B \in \Delta_{k}$ can be easily transformed into 
equivalent restrictions for $A / B$. We omit this detail. The result is:

- If $1 / 8<A / B<\infty$, then $\gamma_{\min }=\gamma_{1}$.

- If $(4(k+1)(k+2))^{-1}<A / B<(4 k(k+1))^{-1}$, then $\gamma_{\min }=\gamma_{k+1}, k=$ $1, \ldots, n-2$.

- If $0<A / B<(4 n(n-1))^{-1}$, then $\gamma_{\min }=\gamma_{n}$.

Our final observation is that we can remove the restriction $2^{m} A+B=1$. Indeed, we have proved inequalities of the form

$$
\left\|f^{\prime}\right\| \leq \frac{A\left\|f^{\prime \prime}\right\|+B\|f\|}{A \theta_{k}+B \mu_{k}} .
$$

The quotient on the right-hand side is homogeneous with respect to $A, B$, so this quotient has the same value for $A, B$ and for $d A, d B$, whatever the positive constant $d$ is.

\section{Acknowledgements}

The research of the first author is supported by the Brazilian Science Foundation FAPESP under Grant 97/11591-5. The research of the second author is supported by the Brazilian Science Foundations CNPq under Grant 300645/95-3 and FAPESP under Grant 97/6280-0.

\section{References}

[1] B.D. Bojanov and A.K. Varma, On a polynomial inequality of Kolmogoroff's type. Proc. Amer. Math. Soc. 124 (1996), 491-496.

[2] G.A. Horn and C.R. Johnson, Matrix Analysis, Cambridge Univ. Press, Cambridge, 1985.

[3] A. Kolmogoroff, On inequalities between the upper bounds of the successive derivatives of an arbitrary function on an infinite interval, in Amer. Math. Soc. Transl. Ser. 1-2, Amer. Math. Soc., Providence, RI, 1962, pp. 233-243.

[4] E. Landau, Einige Ungleichungen für zweimal differenzierbare Funktionen, Proc. London Math. Soc.(2) 13 (1913), 43-49.

[5] A.A. Markov, On a problem of Mendeleev, Zap. Imp. Acad. Nauk., St. Petersburg 62 (1889), 1-24.

[6] V.A. Markov, On functions least deviating from zero on a given interval, St. Petersburg, 1892 [Russian], reprinted in Über Polynome die in einem gegebenen Intervall moglichst wenig von Null abweichen, Math. Ann. 77 (1916), 213-258.

[7] G. Szegö, Orthogonal Polynomials, Amer. Math. Soc. Colloq. Publ., Vol. 23, 4th edn., Amer. Math. Soc., Providence, RI, 1975.

[8] A.K. Varma, A new characterization of Hermite polynomials, J. Approx. Theory 63 (1990), 238-254. 